\title{
The Role of Virtual-Tissue Computer Simulations in the Interpretation of Four-Dimensional Microscopy of Developing Tissues: The Example of Polycystic Kidney Disease
}

\author{
James A. Glazier ${ }^{1}$, Julio Belmonte ${ }^{1}$, Sherry G. Clendenon ${ }^{1}$, J. Scott Gens ${ }^{1}$, Maciej Swat ${ }^{1}$, Abbas \\ Shirinifard ${ }^{1,2}$, Robert Bacallao, ${ }^{3}$ R. Kishore Mosaliganti ${ }^{4}$, Sean G. Megason ${ }^{4}$ \\ 1. Biocomplexity Institute, Department of Physics, Indiana University, Bloomington, Indiana USA \\ 2. Department of Information Sciences, St. Jude Children's Research Hospital, Memphis, Tennessee USA \\ 3. Department of Nephrology, Indiana University School of Medicine USA \\ 4. Department of Systems Biology, Harvard Medical School, Boston, Massachusetts USA
}

Despite having complete genomic sequences for humans and other organisms, we are still far from understanding how an embryo develops with the proper shape, size and pattern. Despite genome-wide association and expression studies, our ability to predict the course of many diseases or to optimize their treatment is limited. One reason for this shortfall is that the organism develops, maintains and fails as a result of physical and molecular control mechanisms interacting via complex patterns of feedback at multiple scales $[1 ; 2]$.

Used properly, mechanistic computer simulations of tissue development based on 3D or 4D microscopy data, known as virtual tissue studies, can assist with hypothesis generation and testing and improve experiment design and interpretation. However, virtual-tissue methods are largely unfamiliar to experimentalists. One reason has been that, until recently, developing virtual tissues required users to learn complex programming methods. In addition, analysis and interpretation of simulation outputs often differed from those familiar in microscopy. As a result, instead of the optimal close coupling between simulation and experiment, information flowed primarily from experiment to simulation. We developed the open-source CompuCell3D software package for multi-cell, multi-scale virtual tissue development, to simplify and accelerate development of virtual tissue simulations by providing standardized tools and high-level specification of molecular-interaction, cell, tissue and organism-level models. We illustrate it using polycystic kidney disease as an example.

Autosomal dominant polycystic kidney disease $(A D P K D)$ is characterized by adult onset and progressive loss of renal function as a consequence of accumulating cysts. About $80 \%$ of ADPKD relates to mutations in polycystin-1, a large integral membrane protein that associates with E-cadherin [3]. While the mechanism by which polycystin-1 acts to drive ADPKD is not known, it seems to involve changes in cell adhesion. Failures in E-cadherin assembly have been implicated on the onset of ADPKD: [4,5]. Polycystin-1 mutants also express cadherin- 8 and ectopic expression of cadherin- 8 in a single cell is sufficient to initiate cystogenesis [6].

Here, using a virtual tissue built using CompuCell3D we investigate how "macroscopic" changes in cell properties like adhesion strength and cadherin localization can lead to ADPKD phenotypes, and what factors may regulate the size and number of cysts. 
We built a virtual tissue ADPKD simulation using the CompuCell3D modelling framework [7]. We modelled the renal tube as a set of virtual epithelial cells composed of apical, basal and lateral surface compartments, and a core cytosolic compartment. We assumed that specification of surface regions depends partially on a cell's external environment, with lateral compartment markers localizing at cell-cell contact interfaces and basal compartment markers localizing at cell-matrix interfaces. In the absence of any external cues, surface compartment markers distribute randomly on the cell surface. On contact, surface markers adhere between cells, colocalizing and activating intracellular signals. Since experiments suggest that contact-inhibition $(C I)$ is likely the main regulator of cell growth in the renal tubule, we employed our own model of CI [8], where the fraction of cell-cell and cell-matrix contact surface regulates epithelial growth. We implemented a simplified model of lumen formation and growth via apical secretion of a viscoelastic fluid regulated by intravesicular pressure.

This simple virtual tissue simulation sufficed to generate a whole cyst from a tubule when a single cell switched from expressing $\mathrm{N}$-cadherin to cadherin-8. The CI model leads the isolated cadherin-8 cell to proliferate. After the first division, the lateral compartment markers of the cadherin- 8 cells co-localize and create a homotypic cell-cell contact surface sufficient to slow, but not halt, proliferation. A lumen forms and the cyst grows until it reaches a typical radius, at which the reduced curvature of the enveloping cadherin-8-expressing epithelium has sufficient CI to halt cell proliferation.

A virtual-tissue simulation of epithelial proliferation, lumen formation and regulation suffices to reproduce significant aspects of cystogenesis in cadherin- 8 induced ADPKD, illustrating the power of virtual tissues in modeling the feedback between structural and biochemical mechanisms in complex developmental diseases.

\section{References:}

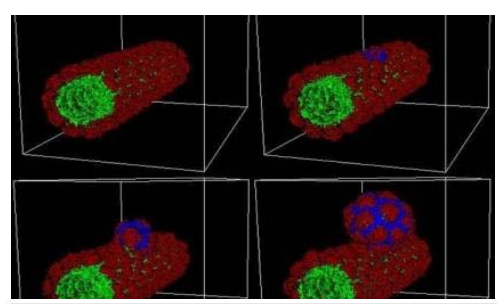

Figure: 3D views of ADPKD simulation. Lumen=green, basal compartment $=$ red and Cadherin-8 expressing lateral compartment $=$ blue; other cell compartments not shown. Cell diameter $\sim 10 \mu \mathrm{m}$. Times, 0h, 4d, 10d, 25d.

[1] GB Blanchard et al., Nature Methods 6 (2009). p. 458-464.

[2] SG Megason et al., Curr. Opin. Genet. Dev. 21 (2011) p. 519-522.

[3] Y Huan, et al., Journal of Clinical Investigation, 104 (1999). p. 1459-1468.

[4] AJ Charron et al., Journal of Cell Biology, 2000. 149(1): p. 111-124.

[5] A Markoff et al., Journal of Molecular Biology, 369 (2007). p. 954-66.

[6] R Kher et al., American Journal of Physiology: Cell Physiology (2011) p. C99-C105.

[7] M Swat et al., Methods in Cell Biology 110 (2012) p. 325-366.

[8] S Jeyaraman et al., PloS Computational Biology (2013) Submitted: PCOMPBIOL-S-13-00178.

[9] The authors acknowledge funding by EPA R-10-0049: The Texas-Indiana Virtual STAR Center, NIGMS R01 GM077138: Competitive Renewal of Development and Improvement of the Tissue Simulation Toolkit and R01 GM76692: Competitive Renewal of Multiscale Studies of Segmentation in Vertebrate Embryos and from Indiana University's Office of the Vice Provost for Research. 